\title{
ABO blood groups and pancreatic cancer risk and survival: Results from the PANcreatic Disease ReseArch (PANDoRA) consortium
}

\author{
COSMERI RIZZATO $^{1 *}$, DANIELE CAMPA ${ }^{1 *}$, RAFFAELE PEZZILLI $^{2}$, PAVEL SOUCEK $^{3}$, \\ WILLIAM GREENHALF ${ }^{4}$, GABRIELE CAPURSO ${ }^{5}$, RENATA TALAR-WOJNAROWSKA ${ }^{6}$, \\ ANETTE HELLER $^{8}$, KRZYSZTOF JAMROZIAK ${ }^{7}$, KAY-TEE KHAW $^{9}$, TIM J. KEY ${ }^{10}$, FRANCO BAMBI ${ }^{11}$, \\ STEFANO LANDI ${ }^{12}$, BEATRICE MOHELNIKOVA-DUCHONOVA ${ }^{3}$, LUDMILA VODICKOVA ${ }^{13}$, \\ MARKUS W. BÜCHLER ${ }^{8}$, PETER BUGERT ${ }^{14}$, PAVEL VODICKA ${ }^{13}$, JOHN P. NEOPTOLEMOS ${ }^{4}$, \\ JENS WERNER $^{8}$, JÖRG D. HOHEISEL ${ }^{1}$, ANDREA S. BAUER ${ }^{1}$, NATHALIA GIESE $^{8}$ and FEDERICO CANZIAN ${ }^{1}$ \\ ${ }^{1}$ Genomic Epidemiology Group, German Cancer Research Center (DKFZ), Heidelberg, Germany; \\ ${ }^{2}$ Department of Digestive Diseases and Internal Medicine Sant'Orsola-Malpighi Hospital, Bologna, Italy; \\ ${ }^{3}$ Department of Toxicogenomics, National Institute of Public Health, Prague, Czech Republic; \\ ${ }^{4}$ Pancreas Biomedical Research Unit and the Liverpool Experimental Cancer Medicine Centre, \\ National Institute for Health Research, Liverpool, UK; ${ }^{5}$ Digestive and Liver Disease Unit, \\ 'Sapienza' University of Rome, Rome, Italy; Departments of ${ }^{6}$ Digestive Tract Diseases and ${ }^{7}$ Hematology, \\ Medical University, Lodz, Poland; ${ }^{8}$ Clinic for General, Visceral and Transplantation Surgery, University of Heidelberg, \\ Heidelberg, Germany; ${ }^{9}$ University of Cambridge School of Clinical Medicine, Cambridge; ${ }^{10}$ Cancer Epidemiology Unit, \\ University of Oxford, Oxford, UK; ${ }^{11}$ Blood Transfusion Service, Azienda Ospedaliero Universitaria Meyer, Florence; \\ ${ }^{12}$ Department of Biology, University of Pisa, Pisa, Italy; ${ }^{13}$ Department of Molecular Biology of Cancer, \\ Institute of Experimental Medicine, Academy of Science of the Czech Republic and First Faculty of Medicine, \\ Charles University in Prague, Czech Republic; ${ }^{14}$ Medical Faculty Mannheim, Institute of Transfusion Medicine and \\ Immunology, Heidelberg University, German Red Cross Blood Service of Baden-Württemberg-Hessen, Mannheim, Germany
}

Received December 21, 2012; Accepted January 24, 2013

DOI: $10.3892 / o r .2013 .2285$

\begin{abstract}
There is strong epidemiologic evidence indicating that common genetic variability could be implicated in pancreatic cancer risk and, to date, various loci have been proposed. In particular, there is increasing evidence of the involvement of $A B O$ gene variability and pancreatic cancer risk. In a large multicentric study of 1,028 pancreatic ductal adenocarcinoma cases and 2,257 controls in the context of the PANcreatic Disease ReseArch (PANDoRA) consortium, we investigated the suggested association with increased risk for carriers of single nucleotide polymorphisms (SNPs) determining the A or $\mathrm{B}$ allele in comparison with the $\mathrm{O}$ allele, which encodes for a non-functional enzyme. Since glycosyltransferase activity, encoded by $A B O$, is higher for the A1 variant compared with
\end{abstract}

Correspondence to: Dr Federico Canzian, Genomic Epidemiology Group, German Cancer Research Center (DKFZ), Im Neuenheimer Feld 280, D-69120 Heidelberg, Germany

E-mail: f.canzian@dkfz.de

*Contributed equally

Key words: pancreatic cancer, $\mathrm{ABO}$, genetic susceptibility, cancer risk the A2 variant, we investigated the hypothesis that A1 carriers were at an increased risk of pancreatic cancer. In our analysis, carriers of the A1 were indeed at greater risk of developing the disease. In addition, we investigated the possible influence that genetic variability at the $A B O$ locus may have in pancreatic cancer survival, but we observed no effect in our population.

\section{Introduction}

Pancreatic cancer is the fourth leading cause of cancer-related mortality in the European Union and in the USA, with a 5-year survival of less than 5\% (1). The latter applies particularly for pancreatic ductal adenocarcinoma (PDAC), the most common form of the disease. There is no effective screening test for pancreatic cancer and metastatic disease is commonly present at initial diagnosis. Established risk factors include cigarette smoking, obesity, a medical history of diabetes type II and family history of pancreatic cancer $(2,3)$. Moreover, it has been shown that a small proportion of pancreatic tumors arises as a result of high penetrance germline mutations in genes such as BRCA1, BRCA2, p16/CDKN2A, STK11/LKB, APC, PRSS1, SPINK, KRAS and PALB2 (2-5). However, the very low frequency of those mutations cannot explain the bulk of genetic susceptibility to pancreatic cancer. 
There is strong epidemiologic evidence indicating that common genetic variability could be implicated in the disease risk and various risk loci have been proposed. Three genomewide association studies have been performed and resulted in several loci convincingly being associated with risk of pancreatic cancer (6-9). In particular, there is increasing evidence of the involvement of the $A B O$ gene variability and pancreatic cancer risk (6,10-12).

The $A B O$ gene encodes enzymes known as glycosyltransferases which transfer specific sugar residues to a precursor substance (the $\mathrm{H}$ antigen) to produce the $\mathrm{A}$ and $\mathrm{B}$ antigens. Glycosylation is one of the most prevalent modifications mediated by complex enzymatic machinery, whereby glycans (sugars) are covalently attached to specific amino acid sites of proteins. Glycans have key biological functions in protein maturation and turnover, cell adhesion and trafficking and receptor binding and activation (13).

There are three major alleles at the $A B O$ locus on chromosome 9q34: alleles A, B and O, defined by single base deletions and substitutions (single nucleotide polymorphisms; SNPs) occurring in exons 6 and 7. The A allele encodes $\alpha 1 \rightarrow 3 \mathrm{~N}$-acetylgalactosaminyltransferase, which adds $\mathrm{N}$-acetylgalactosamine (GalNAc) to the $\mathrm{H}$ antigen to form the A antigen. The B allele encodes $\alpha 1 \rightarrow 3$ galactosyltransferase, which transfers galactose $(\mathrm{Gal})$ to the $\mathrm{H}$ antigen to construct the $\mathrm{B}$ antigen (14). The $\mathrm{O}$ allele does not produce an active enzyme (14). Four SNPs at nucleotides (nt) 526, 703, 796 and 803 resulting in amino acid substitutions (Arg176Gly, Gly235Ser, Leu266Met and Gly268Ala) explain all the differences in the activity and the nucleotide-sugar donor specificity of the A and B transferases. In addition, a base substitution (rs1053878) at nt 467, resulting in an amino acid substitution (proline to leucine), distinguishes the A1 from A2 subtypes. A2 is present in approximately $20 \%$ of subjects with A blood group among Caucasians and shows an intermediate phenotype, between the 'full' enzymatic activity defined by the A1 allele and the nonfunctioning enzyme defined by the $\mathrm{O}$ allele $(11,12)$. The carriers of the A or B allele show an increased risk of pancreatic cancer $(11,12)$. A small number of studies have also tested the genetic variability of the locus with the aggressiveness of the disease and survival of patients, with inconsistent results (15-17).

In this large multicentric study of 1,028 pancreatic cancer cases and 2,257 controls, we replicated the previously reported associations and we also investigated the possible influence of the genetic variability at the $A B O$ locus on survival of patients with pancreatic cancer, considering also the polymorphism discriminating the A1 and A2 variants. Moreover, we performed stratified analysis considering the tumor stage in order to verify whether genetic variability could be involved in the disease prognosis.

\section{Materials and methods}

Study population. The study population was described in detail elsewhere $(18,19)$. Briefly, 1,028 PDAC cases and 2,257 controls were collected retrospectively in five European countries in the context of the PANcreatic Disease ReseArch (PANDoRA) consortium (18). All cases were collected between 1996 and 2011. The cases included in the consortium population are defined by a confirmed diagnosis of PDAC. For each patient, information about gender and age at diagnosis was collected. Detailed information concerning overall survival (OS) was registered as well.

Comprehensive information on the control population was previously provided $(18,19)$. Briefly, a total of 2,121 healthy controls of German, Italian, Czech and Polish origin were respectively recruited at the Institute of Transfusion Medicine, Mannheim, Germany; at the Azienda Ospedaliero-Universitaria Meyer (A.O.U. Meyer) Ospedale Pediatrico, Florence, Italy; at the Department of Molecular Biology of Cancer, Institute of Experimental Medicine, Academy of Science of the Czech Republic and First Faculty of Medicine, Charles University in Prague, Czech Republic; and at the Department of Hematology, Medical University, Lodz, Poland. British controls ( $\mathrm{N}=136)$ were selected from healthy volunteers recruited from the general population in two cohorts in the European Prospective Investigation on Cancer (EPIC), an ongoing prospective cohort being carried out in ten European countries. The EPIC-Norfolk cohort (http://www.srl.cam.ac.uk/epic/) comprises $>30,000$ individuals, aged 45-75 years at recruitment, residents in Norfolk, East Anglia, UK, and recruited from general practice registers between 1993 and 1997 (20). The EPIC-Oxford cohort comprises 65,429 people aged $\geq 20$ years and living in the UK, recruited between 1993 and 1999 (21). Characteristics of patients and controls are provided in Table I.

Selection of genes and polymorphisms. We examined six SNPs on the $A B O$ gene: rs505922, which marks the O allele $(11,12)$, rs8176747, rs8176720, rs8176741, rs8176746, which discriminate between allele $\mathrm{A}$ and $\mathrm{B}$, and rs1053878, which distinguishes between the A1 and A2 alleles. These SNPs account for all the functionally relevant variability at the $A B O$ locus and predict the ABO blood groups, as shown in Table II.

DNA extraction and genotyping. DNA was extracted from whole blood or from frozen or paraffin-embedded pancreatic tissues of patients and controls using the Qiagen-mini kit (Qiagen, Hilden, Germany) or the AllPrep Isolation kit (Qiagen) according to the manufacturer's protocol. Genotyping was performed using an allele-specific PCR-based KASPar SNP genotyping system (KBiosciences, Hoddesdon, UK) as recommended by the manufacturer. The order of DNAs from cases and controls was randomized on PCR plates in order to ensure that an equal number of cases could be analyzed simultaneously. Detection was performed using an ABI PRISM 7900 HT sequence detection system with SDS 2.2 software (Applied Biosystems, Foster City, CA, USA). Genotyping for British controls was performed in the context of a genome-wide association study using the Human 660W-Quad BeadChip array according to manufacturer's instructions (Illumina, San Diego, CA, USA).

Statistical analysis. Hardy-Weinberg equilibrium was tested in the controls by the $\chi^{2}$ test. We used logistic regression for multivariate analyses to assess the main effects of the genetic polymorphisms on pancreatic cancer risk using a co-dominant inheritance model. The most common allele in the controls was assigned as the reference category. All analyses were adjusted for age, gender and country. 


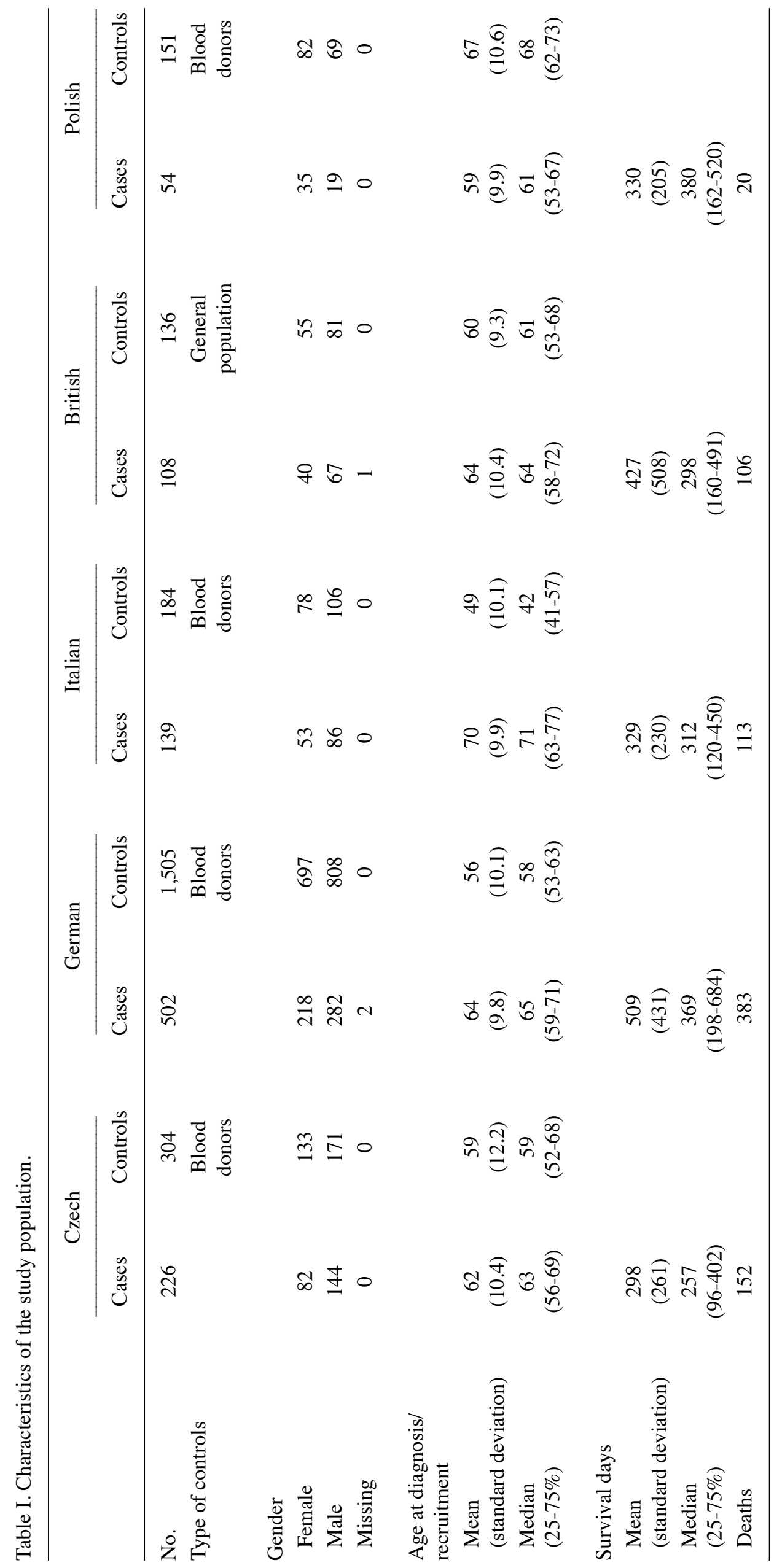


Table II. $A B O$ gene SNP selection.

\begin{tabular}{|c|c|c|c|c|c|c|c|}
\hline \multirow{3}{*}{$\mathrm{cDNA}^{\mathrm{a}}$} & \multirow{3}{*}{$\mathrm{aa}^{\mathrm{b}}$} & \multirow{3}{*}{ SNP } & \multirow{3}{*}{$\mathrm{Tag}^{\mathrm{c}}$} & \multicolumn{4}{|c|}{ Blood group } \\
\hline & & & & $\mathrm{O}$ & $\mathrm{A} 1$ & $\mathrm{~A} 2$ & B \\
\hline & & & & & aa & aa & aa \\
\hline 261 & 87 & rs8176719 & rs505922 & del (frame shift) & & & \\
\hline 293 & 99 & rs8176720 & & & gly & gly & gly \\
\hline 467 & 156 & rs1053878 & & & pro & leu & pro \\
\hline 526 & 176 & rs7853989 & rs8176746 & & $\arg$ & $\arg$ & gly \\
\hline 657 & 219 & rs8176741 & & & his & his & his \\
\hline 703 & 235 & rs8176743 & rs8176746 & & gly & gly & ser \\
\hline 796 & 266 & rs8176746 & & & leu & leu & met \\
\hline 803 & 268 & rs8176747 & & & gly & gly & ala \\
\hline 930 & 268 & rs8176749 & rs8176746 & & leu & leu & leu \\
\hline
\end{tabular}

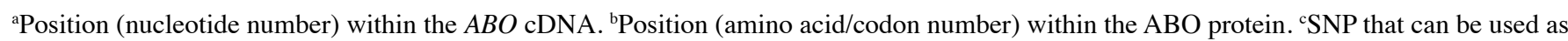
surrogate due to high linkage disequilibrium $\left(r^{2}>0.95\right.$ in HapMap CEU subjects).

For survival analysis, the median follow-up time was computed with censored observations only, whereas the median survival time was calculated using data from all patients. OS was defined as the time interval between diagnosis and death (uncensored observation) or the last date when the patient was still alive (censored observation, mean follow-up time 543 days). OS was evaluated using methods for censored survival time. In particular, risk of mortality was estimated by hazard ratios (HR) and 95\% confidence intervals (CI) in Cox proportional hazard models. All the analyses were performed with STATA software (StataCorp., College Station, TX, USA).

\section{Results}

In this study, we investigated two endpoints: i) to replicate the associations between $6 A B O$ SNPs, predictive of the ABO blood groups, and the risk of developing pancreatic cancer; and ii) to evaluate the possible associations between the same SNPs and patient survival.

We carried out genotyping in 1,029 cases and 2,280 controls. Samples of 1 case and 23 controls were excluded due to low call rates ( $<5$ SNPs successfully genotyped), therefore we performed statistical analyses on 1,028 cases and 2,257 controls. The average call rate of the SNPs was $96.78 \%$ (range 94.48-99.13\%). For 27 cases, both normal and tumor tissues were available and used for genotyping. No differences were observed (398 informative genotype comparisons). Approximately $15 \%$ of the samples were analyzed in duplicate and the concordance rate of the genotypes was $>99 \%$. In controls, the genotype distributions at all loci were within the Hardy-Weinberg equilibrium, with non-significant $\chi^{2}$ values (data not shown).

By examining all the SNPs separately in relation to pancreatic cancer risk, we found that rs8176741, rs8176746 and rs 8176747 were associated with decreased risk of pancreatic cancer, while rs505922 was associated with increased risk of developing the disease. The association of this SNP with pancreatic cancer risk was previously evaluated in a subgroup of the patients of this study and has already been reported (19). The remaining two SNPs (rs1053878 and rs8176720) were not associated with pancreatic cancer risk. The strongest association observed was that with the $\mathrm{T}$ allele of the rs 8176741 SNP $\left(\mathrm{OR}_{\text {het }}=0.73 ; 95 \%\right.$ CI $0.58-0.90 ; \mathrm{OR}_{\text {carriers }}=0.75 ; 95 \% \mathrm{CI}$ : $0.60-0.92)$. The corresponding frequencies and distribution of the genotypes and the odds ratios for the association of each polymorphism with PDAC risk are summarized in Table III. We estimated the risk of pancreatic cancer according to genotype-derived ABO blood type among all the study participants, as shown in Table IV. For 1,035 German controls, information on $\mathrm{ABO}$ status, defined serologically $(\mathrm{O}, \mathrm{A}, \mathrm{AB}$ or $\mathrm{B})$, was collected at recruitment. In $99.3 \%$ of subjects $(1,028 / 1,035)$, the serological blood type and the genotype-derived blood type were identical; this proportion strongly supports the accuracy of the genotype-defined blood group alleles.

In comparison to individuals with O blood type, those with A blood type were at greater risk of developing pancreatic cancer (OR 1.24; 95\% CI 1.04-1.48). Individuals with B or $\mathrm{AB}$ blood types did not show any increase in risk. To address the hypothesis raised by Wolpin et al (12) that the A1 allele confers a greater risk of pancreatic cancer than the A2 allele, we estimated ORs for A1 and A2 alleles compared with O, which was considered as the reference category. By considering all the study population together we observed that individuals with genotype A2/O had OR 1.14 (95\% CI 0.84-1.54), genotype A2/A1 had OR 1.76 (95\% CI 1.08-2.89), genotype A1/O had OR 1.28 (95\% CI 1.06-1.56) and genotype A1/A1 had OR 1.68 (95\% CI 1.14-2.30). Only 2 cases and 7 controls inherited an A2/A2 genotype, severely limiting our ability to accurately assess risk for subjects with this genotype. These analyses are shown in Table IV. When performing subgroup analysis according to country of origin we had comparable results (data not shown). 
Table III. Associations between individual $A B O$ SNPs and risk of PDAC.

\begin{tabular}{|c|c|c|c|c|c|c|c|}
\hline SNP & Genotype & Cases $^{a}$ & Controls $^{\mathrm{a}}$ & $\mathrm{OR}^{\mathrm{b}}$ & $(95 \% \mathrm{CI})^{\mathrm{b}}$ & P-value & Ptrend \\
\hline \multirow[t]{4}{*}{ rs8176720 } & AA & 463 & 974 & & & & 0.168 \\
\hline & $\mathrm{AG}$ & 445 & 1003 & 0.97 & $(0.82-1.14)$ & 0.693 & \\
\hline & GG & 111 & 275 & 0.84 & $(0.65-1.1)$ & 0.205 & \\
\hline & $\mathrm{AG}+\mathrm{GG}$ & & & 0.94 & $(0.8-1.1)$ & 0.443 & \\
\hline \multirow[t]{4}{*}{ rs8176741 } & $\mathrm{CC}$ & 845 & 1689 & & & & 0.009 \\
\hline & CT & 148 & 400 & 0.73 & $(0.58-0.9)$ & 0.004 & \\
\hline & TT & 12 & 27 & 1.07 & $(0.52-2.19)$ & 0.861 & \\
\hline & $\mathrm{CT}+\mathrm{TT}$ & & & 0.75 & $(0.6-0.92)$ & 0.006 & \\
\hline \multirow[t]{4}{*}{ rs8176746 } & $\mathrm{CC}$ & 856 & 1820 & & & & 0.068 \\
\hline & $\mathrm{CA}$ & 158 & 412 & 0.80 & $(0.65-0.99)$ & 0.04 & \\
\hline & AA & 11 & 25 & 1.03 & $(0.49-2.15)$ & 0.948 & \\
\hline & $\mathrm{CA}+\mathrm{AA}$ & & & 0.81 & $(0.66-1)$ & 0.049 & \\
\hline \multirow[t]{4}{*}{ rs8176747 } & GG & 857 & 1697 & & & & 0.027 \\
\hline & $\mathrm{CG}$ & 160 & 405 & 0.77 & $(0.63-0.95)$ & 0.017 & \\
\hline & $\mathrm{CC}$ & 11 & 24 & 1.03 & $(0.48-2.18)$ & 0.946 & \\
\hline & $\mathrm{CG}+\mathrm{GG}$ & & & 0.79 & $(0.64-0.97)$ & 0.022 & \\
\hline \multirow[t]{4}{*}{ rs505922 } & $\mathrm{TT}$ & 342 & 860 & & & & 0.026 \\
\hline & $\mathrm{CT}$ & 518 & 1055 & 1.18 & $(0.99-1.4)$ & 0.066 & \\
\hline & $\mathrm{CC}$ & 163 & 336 & 1.19 & $(0.94-1.51)$ & 0.147 & \\
\hline & $\mathrm{CT}+\mathrm{CC}$ & & & 1.18 & $(1-1.39)$ & 0.048 & \\
\hline \multirow[t]{4}{*}{ rs1053878 } & AA & & & & & & 0.538 \\
\hline & $\mathrm{AG}$ & 866 & 1957 & 1.05 & $(0.84-1.33)$ & 0.659 & \\
\hline & GG & 140 & 285 & 0.58 & $(0.12-2.72)$ & 0.486 & \\
\hline & $\mathrm{AG}+\mathrm{GG}$ & 2 & 9 & 1.04 & $(0.83-1.31)$ & 0.741 & \\
\hline
\end{tabular}

${ }^{\text {a} N u m b e r s ~ m a y ~ n o t ~ a d d ~ u p ~ t o ~} 100 \%$ of subjects due to genotyping failure. All samples that did not give a reliable result in the first round of genotyping were resubmitted to up to two additional rounds of genotyping. Data points that were still not filled after this procedure were left blank. ${ }^{\mathrm{b} O R}$, odds ratio; CI, confidence interval. All analyses were adjusted for age, gender and nationality. Significant associations $(\mathrm{p}<0.05)$ are reported in bold.

Table IV. Associations between blood types determined by 6 SNPs in the $A B O$ gene and risk of PDAC.

\begin{tabular}{|c|c|c|c|c|c|c|}
\hline Blood group & Cases $^{\mathrm{a}}$ & Controls ${ }^{\mathrm{a}}$ & $\mathrm{OR}^{\mathrm{b}}$ & $(95 \% \mathrm{CI})^{\mathrm{b}}$ & $\mathrm{P}$-value & Ptrend \\
\hline $\mathrm{O}$ & 343 & 870 & 1 (ref) & - & - & 0.895 \\
\hline A & 515 & 968 & 1.30 & $(1.09-1.54)$ & 0.003 & \\
\hline $\mathrm{A} 1 / \mathrm{O}$ & 317 & 611 & 1.25 & $(1.02-1.51)$ & 0.028 & \\
\hline A1/A1 & 69 & 112 & 1.57 & $(1.11-2.23)$ & 0.011 & \\
\hline $\mathrm{A} 1 / \mathrm{A} 2$ & 32 & 51 & 1.71 & $(1.04-2.80)$ & 0.033 & \\
\hline $\mathrm{A} 2 / \mathrm{O}$ & 89 & 187 & 1.10 & $(0.82-1.49)$ & 0.517 & \\
\hline $\mathrm{A} 2 / \mathrm{A} 2$ & 2 & 7 & 0.79 & $(0.16-3.87)$ & 0.769 & \\
\hline B & 120 & 295 & 1.04 & $(0.80-1.35)$ & 0.764 & \\
\hline $\mathrm{AB}$ & 48 & 142 & 0.79 & $(0.54-1.14)$ & 0.208 & \\
\hline
\end{tabular}

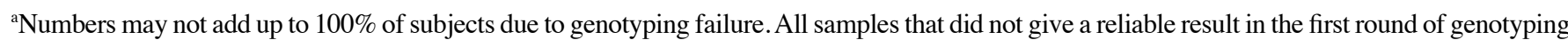
were resubmitted to up to two additional rounds of genotyping. Data points that were still not filled after this procedure were left blank. ${ }^{\mathrm{b}} \mathrm{OR}$, odds ratio; CI, confidence interval. All analyses were adjusted for age, gender and nationality. Significant associations $(\mathrm{p}<0.05)$ are reported in bold. 
Table V. Associations between $A B O$ SNPs and blood types and overall survival of PDAC patients.

\begin{tabular}{|c|c|c|c|c|c|c|}
\hline SNP or blood group & Analysis & Subjects $^{\mathrm{a}}$ & Deaths $^{a}$ & $\mathrm{HR}^{\mathrm{b}}$ & $95 \% \mathrm{CI}^{\mathrm{b}}$ & P-value \\
\hline \multirow[t]{4}{*}{ rs8176720 } & Per allele & 937 & 761 & 0.94 & $(0.84-1.05)$ & 0.264 \\
\hline & AA vs. AG & & & 0.91 & $(0.78-1.06)$ & 0.240 \\
\hline & AA vs. GG & & & 0.91 & $(0.72-1.15)$ & 0.440 \\
\hline & AA vs. (AG+GG) & & & 0.91 & $(0.79-1.05)$ & 0.213 \\
\hline \multirow[t]{4}{*}{ rs8176741 } & Per allele & 922 & 749 & 0.82 & $(0.68-0.99)$ & 0.036 \\
\hline & CC vs. CT & & & 0.79 & $(0.64-0.98)$ & 0.032 \\
\hline & CC vs. TT & & & 0.81 & $(0.44-1.52)$ & 0.521 \\
\hline & $\mathrm{CC}$ vs. $(\mathrm{CT}+\mathrm{TT})$ & & & 0.79 & $(0.65-0.97)$ & 0.027 \\
\hline \multirow[t]{4}{*}{ s8176746 } & Per allele & 941 & 768 & 0.86 & $(0.72-1.03)$ & 0.100 \\
\hline & CC vs. CA & & & 0.86 & $(0.70-1.05)$ & 0.139 \\
\hline & CC vs. AA & & & 0.76 & $(0.39-1.48)$ & 0.422 \\
\hline & $\mathrm{CC}$ vs. $(\mathrm{CA}+\mathrm{AA})$ & & & 0.85 & $(0.70-1.03)$ & 0.105 \\
\hline \multirow[t]{4}{*}{ rs8176747 } & Per allele & 944 & 769 & 0.85 & $(0.71-1.02)$ & 0.077 \\
\hline & GG vs. CG & & & 0.85 & $(0.69-1.04)$ & 0.105 \\
\hline & GG vs. GG & & & 0.76 & $(0.39-1.47)$ & 0.416 \\
\hline & GG vs. (CG+GG) & & & 0.84 & $(0.69-1.02)$ & 0.079 \\
\hline \multirow[t]{4}{*}{ rs505922 } & Per allele & 939 & 763 & 0.98 & $(0.83-1.15)$ & 0.797 \\
\hline & TT vs. CT & & & 1.06 & $(0.85-1.31)$ & 0.610 \\
\hline & TT vs. CC & & & 1.00 & $(0.86-1.16)$ & 0.971 \\
\hline & TT vs. $(\mathrm{CT}+\mathrm{CC})$ & & & 1.02 & $(0.92-1.13)$ & 0.724 \\
\hline A & & 924 & 749 & 1.04 & $(0.89-1.22)$ & 0.604 \\
\hline B & & & & 0.86 & $(0.67-1.10)$ & 0.234 \\
\hline $\mathrm{AB}$ & & & & 0.90 & $(0.62-1.30)$ & 0.577 \\
\hline
\end{tabular}

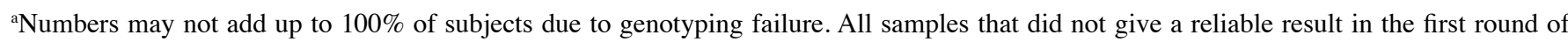
genotyping were resubmitted to up to two additional rounds of genotyping. Data points that were still not filled after this procedure were left blank. ${ }^{b} \mathrm{HR}$, hazard ratio; CI, confidence interval. No association remained statistically significant when multiple testing was taken into account. All analyses were adjusted for age, gender, TNM stage and nationality.

To address the hypothesis that $\mathrm{ABO}$ blood groups may also have prognostic significance, we analyzed the SNPs and the resulting blood groups in relation to patient survival. We found no evidence that genetic variability at this locus modifies the survival of pancreatic cancer, when taking into account multiple testing. We performed stratified analysis considering country of origin and TNM status and we observed no statistically significant association (data not shown). The results of the analysis on the whole consortium are shown in Table V.

\section{Discussion}

Pancreatic cancer is among the most aggressive types of cancer, with mortality rates approaching incidence rates $(1,22,23)$. There is currently no effective curative treatment for pancreatic cancer. Surgery offers the best treatment option and together with some chemotherapeutic treatments it significantly improves survival. Finding genetic variants associated with disease risk is therefore of the utmost importance to define population subgroups who are at high risk of developing the disease. Furthermore, identification of genetic factors associated with progression and survival can significantly contribute to identifying patients with better prognosis and may therefore help clinicians in the choice of treatment options. As there are few known risk factors, improved diagnostics and a better understanding of the molecular pathogenesis of this disease are urgently needed.

In this study, we investigated two endpoints related to ABO glycosyltransferase activity in 1,028 cases and 2,257 controls recruited in the context of the PANDoRA consortium. ABO 
blood group antigens are found in several tissues and in particularly great abundance on epithelial cells. They represent terminal glycosylation steps of glycoproteins or glycolipid (24). Glycoconjugates, such as the ABO antigen, are important mediators of intercellular adhesion and membrane signaling, which are both critical for the progression and spread of malignant cells (25). Moreover, as cell surface molecules they are also recognized by the host immune response and may influence immunosurveillance for malignant cells (26).

Initially, we evaluated the impact of functional genetic polymorphisms on pancreatic cancer risk. We observed that the minor allele of three SNPs (rs8176741, rs8176746 and rs8176747) was associated with decreased risk of pancreatic cancer while rs505922 was associated with increased risk of developing the disease. The remaining two SNPs did not show any association. Polymorphisms rs8176741, rs8176746 and rs8176747 are in high linkage disequilibrium in our population (mean $r^{2}=0.95$ ) and therefore reflect the same association. We typed all of them to have a complete assessment of the phenotypes. By estimating the risk of pancreatic cancer according to the blood type we found that only carriers of the A allele were at increased risk of the disease, while carriers of the $\mathrm{B}$ allele were not. This finding is significant considering the fact that Wolpin et al reported an increase of risk for carriers of A or B allele compared to O carriers (10-12). This is not due to lack of statistical power in our study; we had more than $87 \%$ power to replicate the association with the AB group and more than $99 \%$ for the B group. Our study and the study by Wolpin et al have comparable sizes and the frequencies of the $\mathrm{ABO}$ alleles are similar in the controls, while they differ slightly in the cases (the frequency of $\mathrm{AB}$ was $15 \%$ in PanScan and $10 \%$ in PANDoRA). The discrepancy in the results could be explained by the fact that PanScan individuals were drawn from prospective cohort studies, while PANDoRA subjects were collected from case control studies. Divergent results in cohort and case control studies have already been reported for pancreatic cancer. An example is the association of Sonic Hedgehog ( $\mathrm{SHH}$ ) polymorphisms, associated with increased pancreatic cancer risk in the PanScan prospective cohorts but not in the PanScan case-control studies $(6,8)$.

Wolpin et al (11) suggested that the association of pancreatic cancer with the A allele appears to be predominantly due to the A1 glycosyltransferase, which exhibits higher activity than A2 glycosyltransferase (13). In our analysis, indeed, the less efficient enzyme A2 did not increase the disease risk in comparison with the non-active $\mathrm{O}$ glycosyltransferase.

The results of these two large studies taken together offer a strong direct link between the ABO glycosyltransferase activity and the pathogenesis of pancreatic cancer and suggest that ABO status is not only a marker for pancreatic cancer risk but an actual causative factor for developing the disease. The latter hypothesis remains to be confirmed by functional studies. Since risk factors may also have prognostic significance for pancreatic cancer, as suggested (27), we addressed the hypothesis that $\mathrm{ABO}$ blood types and the genetic variability at the locus could be implicated in patient survival. To date, this has been examined only in studies with a smaller sample size than ours $(16,17)$ with inconsistent results. The samples from Germany partially overlap with those used in a recent study (28). Due to the lack of information on
CA19-9, lymph node status and grading, we were not able to replicate the statistically significant $(0.037)$ association they found using a multivariate analysis. In the univariate analysis, neither study found any statistically significant effect of ABO on survival.

In conclusion, we have confirmed the association of the A blood group with increased risk of pancreatic cancer and we have shown that the risk is mainly due to the A1 allele, establishing a direct connection between the enzymatic activity and the increased risk.

\section{References}

1. Ferlay J, Shin HR, Bray F, Forman D, Mathers C and Parkin DM: Estimates of worldwide burden of cancer in 2008: GLOBOCAN 2008. Int J Cancer 127: 2893-2917, 2010.

2. Anderson K, Mack T and Silverman D: Cancer of the pancreas. Oxford University Press, New York, NY, 2006.

3. Hassan MM, Bondy ML, Wolff RA, et al: Risk factors for pancreatic cancer: case-control study. Am J Gastroenterol 102: 2696-2707, 2007.

4. Jones S, Hruban RH, Kamiyama M, et al: Exomic sequencing identifies PALB2 as a pancreatic cancer susceptibility gene. Science 324: 217, 2009.

5. Tischkowitz MD, Sabbaghian N, Hamel N, et al: Analysis of the gene coding for the BRCA2-interacting protein PALB2 in familial and sporadic pancreatic cancer. Gastroenterology 137: 1183-1186, 2009

6. Amundadottir L, Kraft P, Stolzenberg-Solomon RZ, et al: Genome-wide association study identifies variants in the ABO locus associated with susceptibility to pancreatic cancer. Nat Genet 41: 986-990, 2009.

7. Low SK, Kuchiba A, Zembutsu H, et al: Genome-wide association study of pancreatic cancer in Japanese population. PloS One 5: e11824, 2010.

8. Petersen GM, Amundadottir L, Fuchs CS, et al: A genome-wide association study identifies pancreatic cancer susceptibility loci on chromosomes 13q22.1, 1q32.1 and 5p15.33. Nat Genet 42: 224-228, 2010.

9. Wu C, Miao X, Huang L, et al: Genome-wide association study identifies five loci associated with susceptibility to pancreatic cancer in Chinese populations. Nat Genet 44: 62-66, 2011.

10. Wolpin BM, Chan AT, Hartge P, et al: ABO blood group and the risk of pancreatic cancer. J Natl Cancer Inst 101: 424-431, 2009.

11. Wolpin BM, Kraft P, Gross M, et al: Pancreatic cancer risk and ABO blood group alleles: results from the pancreatic cancer cohort consortium. Cancer Res 70: 1015-1023, 2010.

12. Wolpin BM, Kraft P, Xu M, et al: Variant ABO blood group alleles, secretor status and risk of pancreatic cancer: results from the pancreatic cancer cohort consortium. Cancer Epidemiol Biomarkers Prev 19: 3140-3149, 2010.

13. Moran AP, Gupta A and Joshi L: Sweet-talk: role of host glycosylation in bacterial pathogenesis of the gastrointestinal tract. Gut 60: 1412-1425, 2011.

14. Yip SP: Sequence variation at the human ABO locus. Ann Hum Genet 66: 1-27, 2002.

15. Ben Q, Wang K, Yuan Y and Li Z: Pancreatic cancer incidence and outcome in relation to $\mathrm{ABO}$ blood groups among Han Chinese patients: a case-control study. Int J Cancer 128: 1179-1186, 2011.

16. Dandona M, Gao F, Linehan DC and Wang-Gillam A: Re: ABO blood group and the risk of pancreatic cancer. J Natl Cancer Inst 102: 135-137, 2010.

17. Engin H, Bilir C, Ustun H and Gokmen A: ABO blood group and risk of pancreatic cancer in a Turkish population in Western Blacksea region. Asian Pac J Cancer Prev 13: 131-133, 2012.

18. Campa D, Rizzato C, Capurso G, et al: Genetic susceptibility to pancreatic cancer and its functional characterisation: The PANcreatic Disease ReseArch (PANDoRA) consortium. Dig Liver Dis: Nov 30, 2012 (Epub ahead of print). doi: 10.1016/j. dld.2012.09.014.

19. Rizzato C, Campa D, Giese N, et al: Pancreatic cancer susceptibility loci and their role in survival. PloS One 6: e27921, 2011.

20. Day N, Oakes S, Luben R, et al: EPIC-Norfolk: study design and characteristics of the cohort. European Prospective Investigation of Cancer. Br J Cancer 80 (Suppl 1): 95-103, 1999. 
21. Davey GK, Spencer EA, Appleby PN, Allen NE, Knox KH and Key TJ: EPIC-Oxford: lifestyle characteristics and nutrient intakes in a cohort of 33883 meat-eaters and 31546 non meateaters in the UK. Public Health Nutr 6: 259-269, 2003.

22. Ferlay J, Parkin DM and Steliarova-Foucher E: Estimates of cancer incidence and mortality in Europe in 2008. Eur J Cancer 46: 765-781, 2010

23. Jemal A, Siegel R, Ward E, et al: Cancer statistics, 2008. CA Cancer J Clin 58: 71-96, 2008.

24. Hallouin F, Goupille C, le Cabellec M, Bara J and le Pendu J: Expression of $\mathrm{A}$ and $\mathrm{H}$ blood-group and of CD44 antigens during chemical rat colonic carcinogenesis. Glycoconj J 14: 801-808, 1997.
25. Hakomori S: Antigen structure and genetic basis of histo-blood groups A, B and O: their changes associated with human cancer. Biochim Biophys Acta 1473: 247-266, 1999.

26. Hakomori S: Tumor-associated carbohydrate antigens defining tumor malignancy: basis for development of anti-cancer vaccines. Adv Exp Med Biol 491: 369-402, 2001.

27. Li D, Morris JS, Liu J, et al: Body mass index and risk, age of onset and survival in patients with pancreatic cancer. JAMA 301: 2553-2562, 2009.

28. Rahbari NN, Bork U, Hinz U, et al: AB0 blood group and prognosis in patients with pancreatic cancer. BMC Cancer 12: 319, 2012. 